\title{
Authentication, scale-relativity, and relational kindhood
}

\author{
Ana-Maria Creţu ${ }^{1}$
}

Received: 19 August 2020 / Accepted: 4 January 2022 / Published online: 27 February 2022

(c) The Author(s) 2022

\begin{abstract}
This paper proposes a new natural kinds framework according to which kindhood is relational, dynamic, and scale-relative. Reflecting on the ontogenesis of a scientific classification, I argue that there are two distinct conceptual stages to a scientific classification: a first stage in which enough entities and relations must be authenticated for kindhood to emerge and a second in which the nature of authenticated entities and relations is investigated. The new framework is scale-relative and explains both the changing nature of the entities and relations themselves as well as the changing nature of the classifications in which they are organised.
\end{abstract}

Keywords Authentication - Scale-relativity $\cdot$ Relational kindhood · Natural kinds · Perspectives $\cdot$ Research traditions $\cdot$ Numerosity

\begin{abstract}
This paper has been a long time in the making and has gone through several complete re-writings. Along the way, I had many useful conversations with friends and colleagues, notably, Karim Thébault, Nicos Stylianou, Vanessa Seifert, James Ladyman, Don Ross, Stephan Hartmann, Richard Dawid, Peter Evans, Franklin Jacoby, and Max Jones. Special thanks go to Karim Thébault and Nicos Stylianou who read and commented on several different drafts of this paper. Michela Massimi, Joe Dewhurst, Peter Evans,

Vanessa Seifert, James Ladyman, and Alexander Franklin have also read one of the earlier versions of this paper. Discussions with Vanessa Seifert and James Ladyman have helped immensely in re-canvassing and fine-graining some of my earlier thoughts on real patterns and scale-relativity. Several anonymous reviewers have also offered comments which have ultimately driven me to re-write this final version of the paper. Finally, thanks to audiences at Bristol, Dubrovnik, Exeter, Salzburg, Durham, and Munich for very helpful comments on earlier versions of this paper. Work on this article has been partially undertaken whilst working on a project that has received funding from the European Research Council (ERC) under the European Union's Horizon 2020 research and innovation programme (grant agreement European Consolidator Grant H2020-ERC-2014-CoG 647272 Perspectival Realism. Science, Knowledge, and Truth from a Human Vantage Point).
\end{abstract}

\footnotetext{
$凶 \quad$ Ana-Maria Creţu

d.cretuanamaria@gmail.com

1 University of Bristol, Bristol, UK
} 


\section{Contents}

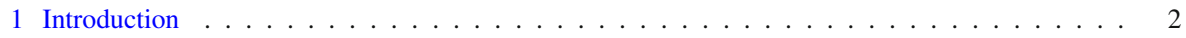

2 Authentication and perspectival development . . . . . . . . . . . . . . 4

2.1 Authentication . . . . . . . . . . . . . . . . . . . . 5

2.2 Perspectival development . . . . . . . . . . . . . . . . . . . . . . 11

3 Relational kindhood and scale-relativity . . . . . . . . . . . . . . . . . . . . 13

3.1 Scale matters . . . . . . . . . . . . . . . . . . . . . . . . . 13

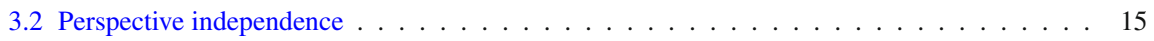

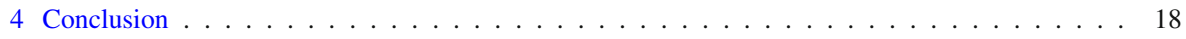

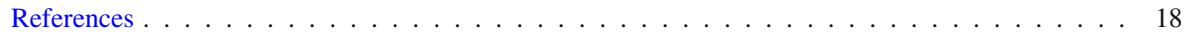

\section{Introduction}

Cats don't exist at the quantum scale and mountains don't exist at astrophysical scales (see Ladyman and Ross 2007); the size of a material can make it or unmake it a nanomaterial (see Bursten 2016); chemical elements can be said to exist only if they live for at least $10^{-14} \mathrm{~s}$; periodical cicadas emerge only every 13 or 17 years; the mechanism for neutrino-mass generation may only obtain at the very highest of energy scales, and multi-scale systems, such as bones or steel, exhibit different properties at different scales (see Wilson 2017; Batterman and Green 2020; Hendry 2021). All these examples point to the scale-relativity of ontology, a thesis articulated and developed as early as 2007 by Ladyman and Ross, according to which what exists should be indexed at relevant scales of measurement, such as length, energy, time, space, numerosity, and so on. Yet, given the significance and ubiquity of scale-relativity in science, it is surprising that explicit discussions of the implications of this thesis for the ontology and methodology of scientific classifications still constitute the exception rather than the norm.

This paper investigates the role and implications of scale-relativity for scientific classifications by looking into their ontogenesis, that is by analysing how scientific classifications come about and how they evolve. Through this investigation I ultimately aim to establish two theses: i) that enough entities and relations must be authenticated to even begin to speak of classifications and ii) that kindhood is relational, dynamic, and scale-relative.

The first thesis concerns the early stages of a classification and emphasises the foundational role of the as yet underappreciated numerosity scale. Numerosity concerns the number of entities ${ }^{1}$ needed to erect a classification and the classificatory changes that occur with changes in quantities. The basic idea behind numerosity is that enough entities or some relation presumed to hold amongst particular entities must be authenticated to begin to talk about kinds.

Authentication refers to the identification and validation of a particular empirical phenomenon as genuine, and it is crucial to classification since no classification can

1 Entities, as explained below are a variety of authenticated phenomena. Entities and relata are used interchangeably throughout, albeit in a way that does not make any deep metaphysical assumptions about their nature. 
be erected on the basis of spurious phenomena. ${ }^{2}$ It will be shown that phenomena can only be authenticated in relation to other phenomena which means that some form of relational background must precede authentication. On the basis of ontogenetic considerations, it will also be shown that phenomena must be authenticated in terms of either entities or relations.

To motivate and unpack this first thesis we will turn to Laudan's (1977) work. In particular, the crucial distinction between 'research traditions' and 'perspectives', adapted from Laudan, will serve to explain how entities and their relations are authenticated and how relational kindhood evolves. Following Laudan (1977), a research tradition is understood as "a set of assumptions: assumptions about the basic kinds of entities in the world, assumptions about how those entities interact, assumptions about the proper methods to use for constructing and testing theories about those entities" (p. 97). In contrast, the notion of 'perspective' is used to mean a more sophisticated and fine-grained theoretical framework that encompasses the set of theoretical interests and background theoretical knowledge that a researcher or group of researchers can be said to hold at any given time. ${ }^{3}$ This conceptual distinction will ultimately serve to show what kind of ontology can be justified within a complex theoretical framework, once the complex layers of the theoretical framework are understood and justified.

It will be argued that entities and their relations are usually authenticated prior to the development of perspectives on their nature. ${ }^{4}$ It will ultimately be established that a research tradition affords the authentication of empirically genuine entities and relations, whilst perspectives are developed to study their precise nature in terms of their origin, constitution, or evolution. To the extent to which the distinction between research traditions and perspectives is accepted, it will be argued that entities and their relations are perspective-independent empirical phenomena. As authenticated phenomena, independent of perspectives, the commitment to entities and their relations will be shown to constitute a legitimate ontological commitment for a natural kinds account.

The second thesis concerns the evolution of a scientific classification. It will be argued that by understanding kindhood as relational, dynamic, and scale-relative we can account for both the changing nature of the phenomena themselves as well as the changing nature of the classifications in which they are organised. To establish the second thesis, the methodology of authentication will be further grounded in the history and philosophy of science through Whewell's (1837a, 1837b, 1837c) history and Whewell's (1840a, 1840b) philosophy of classificatory sciences, which constitutes the first extended modern survey of the methodology and ontology of inductive sciences. In particular, Whewellian considerations will serve to illustrate the interplay between the authentication of phenomena and the application of classificatory principles for their hierarchical organisation and evolution.

\footnotetext{
2 Hacking (1983) makes a similar remark in relation to experiment: "A completely mindless tampering with nature with no understanding or ability to interpret the result, would teach almost nothing" (p. 153).

3 This is similar to Laudan's 'theory'. For a different conception of perspectives see Massimi (2018a). On finer grained notions of 'perspective', both broader and narrower, see Creţu (2021).

4 There can be cases where a novel entity may be predicted in the context of a perspective which gives at least a partial account of the entity's nature; yet even in those cases authentication and perspectival development will play different roles, as will be shown throughout.
} 
Ontogenetic considerations and Whewellian historical lessons will be ultimately used to show that classifications emerge with empirically driven authenticated relations and entities and evolve with the development of perspectives which are continuously informed and reinforced through 'unintermitting' observations of scale-relative empirical phenomena.

There are two novel aspects of this paper. First, it constitutes an in-depth exploration of scale-relativity and especially of previously underappreciated scales such as numerosity and of the interaction between different scales. Second, the paper introduces ontogenetic considerations to separate two distinct conceptual stages of scientific classifications which play very different roles, i.e., authentication and perspectival development.

The present account is first and foremost informed by views on scale-relativity proposed by Ladyman and Ross (2007) and Bursten (2016). ${ }^{5}$ A pivotal role is also played by Laudan's (1977) account of progress in science, Hacking's (1983) and other New Experimentalists ${ }^{6}$ views on experiment and observation in science, and most importantly, the many ideas on the nature of scientific classifications present in Whewell's (1837a, 1837b, 1837c, 1840a, 1840b) extended survey of the ontology and methodology of scientific classifications. These accounts have been invaluable in shaping the original framework proposed in this paper. The present account has also been influenced by Ladyman and Ross's (2007) real patterns based account of natural kinds as well as by Boyd's (1991, 1999a, 1999b) homeostatic property cluster kinds and cognate naturalistic accounts such as Magnus's (2012), Massimi's (2014), and Slater's (2015). The resulting account can be situated within the so-called 'practice and history oriented shift', most recently exemplified in the various proposals found in Kendig (2016b), and stemming from earlier work in a range of fields begun in mid-70's (see Soler et al. 2014).

The paper is structured as follows: Section 2 explains how scientific classifications emerge by establishing a principled conceptual distinction between the authentication stage and the perspectival development stage. Section 3 describes the evolution of scientific classifications by showing how the interplay between the authentication of entities and relations and the application of classificatory principles gives rise to dynamic, scale-relative, relational kindhood. Conclusions follow in Sect. 4.

\section{Authentication and perspectival development}

In their Standford Encyclopaedia article on natural kinds, Bird and Tobin (2017) offer the Standard Model classification of fundamental particles as one of the paradigmatic examples of natural kinds. They claim that " $[\mathrm{t}]$ he standard model in quantum physics reveals many kinds of fundamental particles (electron, tau neutrino, charm quark), plus broader categories such as kinds of kind (lepton, quark) and higher kinds (fermion,

\footnotetext{
5 The views of Dennett (1991), Ross (1995), and Wallace (2010) on real patterns have also been invaluable in shaping the present account.

6 A systematic philosophy of experiment, i.e. the New Experimentalism, begun to emerge in the 80's with the works of Hacking (1983), Cartwright (1983), Ackermann (1985), Franklin (1986), Galison (1997), and others. These, as well as more recent works, played a substantial role in shaping the author's views.
} 
boson)". The Chemical Table of Elements is another 'paradigmatic' example of a successful scientific classification. The rigid structure of The Standard Model and The Periodic Table, in their final forms, provide a Procrustean bed, which all other classifications are expected to fit. Yet a closer look at how these classifications were designed and how they evolved, and continue to evolve, reveals both a marked neglect of scale-relativity in these discussions, as well as associated problems therewith.

For example, The Periodic Table of Elements can be said to be vulnerable to a 'scaling problem', caused by some superheavy elements ( $Z=119$ onwards). Such elements may turn out to threaten the periodicity of elements which constitutes the periodic table's organising principle (Ball 2019). This is because the lifetime of superheavy elements with atomic number higher than 119 is too short for them to acquire outer electrons which make them have chemical properties and thus count as chemical elements (see Kragh 2017 for details). Similarly, discoveries relating to neutrino mass may be said to point to a 'scaling problem' for The Standard Model too, since "the mechanism for neutrino-mass generation and its energy scale" (Rayner 2020) is not yet known, but may point to "physics at a very high energy scale such as the Grand Unification of elementary particle interactions" (Kajita 2015, p. 21).

The main point to emphasise in connection to these examples is not that these classifications aren't outstandingly empirically successful, but rather that they are evolving classifications, with no rigid structure or final form. Another related point to emphasise here is that valuable lessons about the methodology and ontology of scientific classifications can be lost by neglecting scale-relativity and the ontogenesis of scientific classifications. Though such lessons have not been lost on philosophers of biology or biologists who focus on scientific classifications ${ }^{7}$, on philosophers of chemistry with a historicist bent (e.g. Scerri 1998, 2019; Chang 2016), or historians of science (e.g. Kragh 2013, 2017, 2018, 2019), they have not yet become the mainstay of natural kinds debates. ${ }^{8}$

In what follows the role and implications of scale-relativity for scientific classifications will be investigated. Scale-relativity will be shown to have both an ontological and a corresponding methodological dimension and to play different roles at different stages of classificatory development. In particular, two conceptually distinct stages will be delineated: authentication and perspectival development. The first stage, as we shall see, concerns the validation of a phenomenon as empirically genuine and plays a distinct role for the ontology of scientific classifications, whilst the second stage will be shown to be crucial for understanding the precise nature of any phenomena.

\subsection{Authentication}

Authentication refers to the stabilisation and validation of phenomena and is necessary in order to eliminate, insofar as it is possible, potential errors due to

\footnotetext{
7 The relation between evolution and scientific classifications has been the focus of heated debates in biology and philosophy of biology during the '70 and '80, see Kearney (2007) and Richards (2016) for helpful overviews and Sober (2000) for a standard reference.

8 Some natural kinds debates continue to centre on outmoded Kripkean and Putnamiam ideas about kinds, see for example the recent Synthese Special Issue on Natural Kinds: Language, Science, and Metaphysics, Moreno (2019).
} 
experimentation, measurements, or "freak results" (p. 18) and in order to resolve disagreements. ${ }^{9}$ Authentication consists of both a theoretical and empirical component but it is ultimately about phenomena. Authentication is required in connection to any new phenomena, at any stage within the development of a classification. The theoretical side of authentication does not presuppose full elucidation of the nature of the phenomena in question. The phenomena must nonetheless be embedded within a scientific theory which has, at least in principle, physical significance and some degree of relational coherence with cognate theories. The theoretical and empirical components are important for the following reasons:

i. because a phenomenon can be theoretically validated or embedded within a scientific theory but not be experimentally validated (e.g. strings or super superheavy elements past $\mathrm{Z}=119$, the Higgs Boson prior to 2012);

ii. because a phenomenon can be empirically validated, but not theoretically validated (e.g. the positron between 1931 and 1933, plausibly dark matter);

iii. because a phenomenon can be partially authenticated (e.g. the authentication of the Quaking Aspen as an individual tree rather than as a clonal colony);

iv. because a phenomenon can be mis-authenticated. For example, the infamous OPERA superluminal neutrinos, although initially experimentally validated were subsequently shown to have been mis-authenticated due to measurement errors (see Reich 2011; Brumfiel 2012). ${ }^{10}$

Authentication then can be neither purely theoretical nor purely empirical. Authentication plays a foundational role in the incipient stage of a scientific classification but it continues to play a significant role throughout its development too. ${ }^{11}$ Authentication determines that a phenomenon is; perspectival development determines what it is.

Authentication can only be achieved within a research tradition, against a certain background. A research tradition, following Laudan (1977) can be understood as a set of assumptions about what basic entities might there be in the world, how such entities might interact, and how they might be studied. A research tradition is not static and over time can become very specialised and complex. Thus, depending on the phenomenon at hand, the research tradition which studies it and its stage of development, authentication may involve minimal assumptions about the nature of the world or a more complex network of assumptions. For example, the most common assumptions in connection to scientific classifications are that the world is structured, that it is stable enough to be amenable to study and observation, that some entities can be grouped together so as to enable significant generalisations, that entities can be organised in hierarchies etc. ${ }^{12}$ In the case of more advanced research traditions, such as The Standard Model, complex assumptions enter the authentication process and perspectives can become involved in the process too. Note, however, that even in such cases a distinct conceptual role is played by those perspectives that enter into the authentication

\footnotetext{
9 See Creţu (2020) for the authentication of the positron and attending disagreements.

10 For more examples of allegedly incontrovertible empirical facts which turned out not to be authentic phenomena, see Bondi (1955).

11 Authentication implies, though is not reducible to, the empirical confirmation of a phenomena.

12 This point about assumptions is extensively made by Kant (1781), see especially the Appendix pp. 590-604, and also by Whewell (1840a), see especially pp. 18-41.
} 
process and those which concern the development of theories regarding the nature of the authenticated phenomena. That is to say that the origin, evolution, and constitution of any phenomena can be properly investigated only once the phenomena are validated and made amenable to further investigation. ${ }^{13}$ For example, chemical elements past $\mathrm{Z}=119$ cannot as yet, if at all, be stabilised for the time required to acquire outer electrons which would enable further investigation into their nature; such elements then are not as yet authenticated.

It is further worth emphasizing that authentication can be a lengthy and complex process. The Standard Model contains more than one example of lengthy and complex authentication. The authentication of the neutrino constitutes one such example. The neutrino was predicted in 1930 as an essentially massless particle to resolve particular anomalies in $\beta-$ decay, it was first detected in 1956, decades later was found to have mass, and its status is still not fully resolved [see Brown (1978), Kajita (2015), Hernandez (2016), and Rayner (2020) for details]. ${ }^{14}$ Another example concerns the classification of the positron which involved a drawn out process of authentication prior to further investigations into its nature (Creţu 2020; Roqué 1997; Darrigol 1988; Hanson 1961, 1962).

The case of the positron is significant for making another observation in connection to authentication, namely that any new phenomenon is authenticated in relation to other phenomena. For example, the positron was authenticated as a new particle only in relation to and by comparison with other existing particles, i.e., the electron, the proton, and the neutron. This is not to say that the authentication of the positron was an entirely theoretical matter. Nor is it to say that perspectives on the nature of the positron were not developed prior to its authentication. Rather, the claim is that we can only profess to have understood the nature of a phenomenon if the phenomenon is genuine, that is, if it has been authenticated as a genuine phenomenon. Only then can its nature be precisely determined. The distinction between authentication and perspectival development is precisely aimed to capture these two distinct conceptual stages in the classification and study of phenomena.

Two more examples will further serve to highlight the importance of authentication as a distinct conceptual stage in the development of a scientific classification as well as its drawn out character. A first case in point concerns the emergence of the first modern astrophysical classifications which, similarly to the positron case, was also marked by a relatively drawn out authentication. Fat, thin, and fluted patterns on the spectroscopic photographs were authenticated without prior knowledge of the information contained within them (see Cannon and Pickering 1901; Russell 1919; Hoffleit 1991). These authenticated patterns, revealed in the spectra of stars constituted the basis of the first three instalments of the The Henry Draper Catalogue, the third instalment being internationally adopted in 1910. With some modifications, the third instalment of The Henry Draper Catalogue is still in use today. Investigations into the nature of stars emerged only after the authentication and classification of stars based on their spectral characteristics.

\footnotetext{
13 Feest (2011) offers similar suggestions.

14 Hoefer and Martì (2020) also discuss the lengthy process of establishing the reference of the neutrino whilst also suggesting that The Standard Model physics should be placed in a 'quarantine' zone-a zone which does not yet give rise to scientific 'lore' or a core of scientific truths.
} 
A second case in point comes once more from The Standard Model and concerns its most recent addition, the Higgs Boson. Though popular accounts are wont to offer definite pronouncements in relation to the 'discovery' of the Higgs Boson as a singular event, a closer look reveals in fact a relatively complex authentication process. To be precise, the historical details show that the July 2012 discovery was a discovery of a "Higgs-like particle" and neither the CMS nor the ATLAS discovery papers claimed to have definitely discovered the Higgs Boson, but only to have discovered a new boson (see Franklin 2017). ${ }^{15}$ In an analysis of the discovery, Dawid (2015) pointed out the ongoing authentication of the Higgs Boson ${ }^{16}$ and recently Mättig and Stöltzner (2019) showed that "[w]ith the growing evidence that the newly discovered particle has properties consistent with the SM expectations, most physicists accepted it to be a Higgs, and at least tentatively, a SM Higgs" (p. 93). Besides its relatively complex authentication, what the case of the Higgs Boson illustrates is that discovery does not equal authentication. For a phenomenon to count as fully authenticated it must be both experimentally and theoretically validated. Note, however, that theoretical validation does not imply a full elucidation of the precise nature of the phenomena.

Before we move on to the perspectival development stage, some clarifications on the ontology and methodology of authenticated phenomena are in order. We have thus far talked about the authentication of phenomena, but classification involves a finergrained ontology than the ontology of phenomena. A minimal ontology of scientific classifications comprising relations and relata is motivated in what follows on the basis of ontogenetic considerations.

To begin with, historical practices of classification, as described in Whewell's History and Philosophy of the Inductive Sciences, suggest that the authentication of entities traditionally preceded the authentication of relations. In particular, as Whewell (1840a) notes in The Philosophy of the Inductive Sciences, “... before we can attend to several entities as like or unlike, we must be able to apprehend each of these by itself as one thing" (p. 449). To use Whewell's example, the basic idea here is that to be able to talk about a tree, in a forest of trees, we must apprehend the tree as one unit, with its own trunk, branches, leaves and so on. Only once we have thus singled out or authenticated each tree, we can attend to what is alike and what is different amongst the trees.

Yet, the converse is equally true of scientific practice. For instance, the aforementioned example concerning astrophysical classifications, shows that the spectral characteristics of stars, which denote relations amongst stars, were authenticated prior to the authentication of individual stars per se. To be precise, classifications were designed on the basis of their spectral lines, initially based on the strength of the lines and later width, flutedness, and haziness also became relevant. The strength of the spectral lines signifies the temperature of a star; the width can be correlated with the luminosity of a star, whilst the shape of the line can offer information about the atmosphere of a star (see Green and Jones 2015 for more details).

\footnotetext{
15 "Both groups promised a more rigorous test of their conclusions and a further search for physics beyond the SM. Both the conclusion and the title of the papers claimed the discovery of a new boson, but neither definitely claimed that it was the Higgs boson" (Franklin 2017, pp. 272-273).

16 Dawid (2015) cautioned that "[i]t remains to be seen whether the discovered particle has the properties predicted by the standard model of particles physics or must be understood in terms of an extension of the standard model such as supersymmetry", p. 76.
} 
What the examples above indicate is that depending on the scientific practice at hand, it will be a contingent matter whether we attend to the authentication of the relations or of the entities first. Note that on the present account, further specifications of what entities are and to what extent, if at all, they can be further decomposed can only be done on a case by case basis, once perspectives onto their nature have been developed. ${ }^{17}$ Entities, here, are those items which classificatory systems, in different sciences, seek to group into kinds and higher hierarchies. Such entities may on occasion be both new items whose status is provisionally debated, items whose reality is not outright contested, or items that are judged by common sense to be real entities.

Recognising the importance of both entities and relations as the basis of any classification has the added advantage of removing unnecessary limitations to the domain of applicability of scientific classifications. For example, Ladyman and Ross (2007) take natural kinds to be inapplicable to quantum contexts where all entities such as electrons possess the same properties, i.e., rest mass, charge, spin, and are thus intrinsically indistinguishable from one another. ${ }^{18}$ Since natural kinds classifications require that entities differ in their detailed characteristics whilst sharing other characteristics, natural kinds frameworks seem inappropriate in situations such as the quantum context, where all entities share all the same intrinsic characteristics. ${ }^{19}$ However, if it is possible to distinguish a class of identical entities from another class of identical entities, the basis of a classification becomes once again discernible. Thus, whilst all electrons are defined by particular quantum numbers, the electron's quantum numbers differ from the quantum numbers of other quantum particles. That is to say, the electron is a distinct kind from the positron say, precisely because the two differ in some of their characteristics and have been authenticated as different entities. It seems then that the individuality issue in quantum mechanics concerns the nature of phenomena and not the classification of quantum phenomena as such. ${ }^{20}$

This brings us to another point worth highlighting, namely that authentication is not individuation. ${ }^{21}$ Individuation concerns the metaphysical nature of the phenomena which seems to require knowledge of the phenomena that can only be obtained after significant progress in the understanding of their nature has been undertaken. But, as the above examples indicate, phenomena can be at least partially authenticated without the adoption of an explicit stance on their individuality. For example, whilst the individuality or not of 'quantum particles' is still a matter of intense debate amongst philosophers, we have nonetheless been able to authenticate electrons and made significant progress in their study. Similarly, in the case of the forest tree species Quaking Aspen, it seems that despite a raging ongoing debate on whether it is best to count it as a ramet (a single stem or module) or as a genet (a grove or a clone), we have

\footnotetext{
17 A similar point, albeit within a different framework is also made by Reydon (2016).

18 Ladyman (2007, 2015) defends a form of weak discernibility compatible with a relational approach to quantum particles, an approach that is congenial to the present account.

19 Thanks to Richard Dawid for discussion on this point.

20 For an overview of this discussion see French (2019); for further discussions see Ladyman and Ross (2007, ch.3), Muller and Saunders (2008) and Caulton (2013).

21 The present account is nonetheless compatible with forms of 'practical individuation' and 'epistemic individuation', for details see Bueno et al. (2018).
} 
nonetheless been able to at least partially authenticate it and made significant progress in understanding its nature. ${ }^{22}$

A final point worth emphasising in relation to the authentication of entities and relata is that a pronouncement on the ontological priority of entities over relations or of relations over entities seems to be unnecessary when it comes to scientific classifications. There are at least three related reasons why this is so. First, at any given scale, both entities and their relations lie at the heart of classificatory systems across the sciences, synchronically and diachronically. For this reason, any account that is committed to practice-relativity, must embrace both entities and their relations as on a par, on a given scale. Second, for the kind of epistemic purposes classifications are designed to fulfil, there is an important sense in which no classification is more fundamental than another. ${ }^{23}$ Given this and the fact that any classification begins and grows with the authentication of entities and relations amongst them, there's little, if anything to gain from eliminating one or the other or from trying to reduce one to the other. Third, neither entities, nor relations subsist. To be precise, within a specified scientific domain, on large enough timescales there are no entities and on small enough timescales there are no relations, and hence no kinds. Moreover, depending on how extended the timescale is, the same entity may be regarded as either an object, an event, or a process. For example, on timescales small enough, certain supernovae can be regarded as stellar explosion events. On timescales that track the evolution of a star from progenitor to explosion, the supernovae can be regarded as one object that naturally evolves towards its explosion, just as humans naturally evolve to then eventually die. Furthermore, on an even lengthier timescale, supernovae can be regarded as particular types of astrophysical processes. ${ }^{24}$ For these reasons, the present account is committed to the fact that both relations and relata are necessary for purposes of classification $^{25}$ and neither can be eliminated in a truly practice and scale-relative framework.

We have thus far shown that no classification can proceed without authentication. But authenticated phenomena change or evolve (ontological evolution) and the classifications that track the phenomena must change accordingly (methodological and conceptual evolution), though not necessarily, or even usually, simultaneously. To understand how this happens we'll need to analyse the perspectival development stage of classifications before we move on to a more detailed analysis of scale-relativity in Sect. 3 .

\footnotetext{
22 For details on the individuation of plants, see Clarke (2010, 2012). For details on the natural history of the Quaking Aspen see Mitton and Grant (1996).

23 For a related discussion about the purposes of classifications, and a relational approach to kindhood, see the excellent paper of Okasha (2002).

24 For details on the evolution of supernovae see Zeilik and Gregory (1998) and Green and Jones (2015).

25 The present view is thus compatible with three distinct views concerning the relative priority of relations and relata: (a) a view on which relations are primary and entities are secondary, see Stachel's (2006); (b) a view on which entities are primary and relations are secondary, see Russell (1911); and (c) a view on which neither entities nor relations are primary, see Esfeld and Lam (2008), Pooley (2006).
} 


\subsection{Perspectival development}

This section aims to establish that no classification can grow and evolve without understanding the nature of authenticated phenomena. Such understanding, as we shall see, requires the constant development of 'perspectives' on the nature of the phenomena. It is perspectives, and not research traditions, that particularise the ontology of nature. Before we look at some examples, let us first say more about perspectives.

Perspectives are sophisticated theoretical frameworks that encompass the set of theoretical interests and background theoretical knowledge that a researcher or group of researchers can be said to hold at any given time. They have a narrower scope than research traditions and are thus more specialised and fine grained. But, since they are sponsored by research traditions, they also inherit a large proportion of the assumptions of the research tradition.

The role of perspectives is to determine, to the extent to which it is possible, the precise nature of the authenticated phenomena. The establishment of perspectives thus relates to the interpretation of the phenomena. Since phenomena, even authenticated phenomena, can generally underdetermine their interpretation, to determine the precise nature of the phenomena, post-authentication perspectival development is necessary. Perspectives must be compatible with and applicable to the empirical phenomena, explanatory, and at least in principle testable. It may be the case that, at the level of perspectives, the interplay between authenticated empirical phenomena and theoretical principles of classification is overall less independent from anthropocentric concerns. For this reason it is important to emphasise that authentication within a research tradition is largely independent of perspectives (a point we will return to towards the end of the next section). ${ }^{26}$

To illustrate, consider once again the case of the Higgs Boson. One might think that the existence and theoretical role of the SM Higgs Boson, as well as its nature (properties, interactions, etc.) were clear to physicists long before the Higgs Boson was eventually discovered in July 2012 and that physicists knew well what they were looking for before they actually found it.

However, a closer look at the history of the Higgs Boson reveals a similar structure to the one described above, namely that perspectival development on the nature of the Higgs Boson followed authentication. More importantly, the details of the Higgs also highlight two other important lessons: that the theoretical nature of an entity may or may not correspond to the empirical nature of the entity and that discovery does not equal authentication (a point already emphasised). The history of the Higgs search and discovery shows that the determination of the energy range within which the mass mechanism of the Higgs could be found involved a host of complex arguments, strategies, and indirect evidence [see Dawid (2015) and Franklin (2017)]. What this means is that physicists knew only roughly what/where to look for. It would thus be inaccurate to claim that having a prediction and knowing roughly what the energy range where a Higgs like particle could be found amounts to knowledge of the nature (properties, interactions, etc.) of the SM Higgs Boson. It is not the case that the precise properties

\footnotetext{
26 Note that perspective-independent authentication does not imply 'theory-neutral observation of the world', since a research tradition is after all a theoretical framework or 'perspective' itself (albeit a rather broad one).
} 
of the SM Higgs Boson were known before its authentication or the perspectival development stage. Rather, as Mättig and Stöltzner (2019) point out, in 2011, "physicists were rather undecided whether the SM Higgs Boson would eventually be found" (p. 74) and expected that "finding a particle consistent with the SM Higgs would only be the first step in further investigating the properties of the new particle" (p. 80). Even after the discovery of a 'Higgs-like' particle was announced in 2012, physicists would not even claim that it was definitely the SM Higgs Boson, much less that they knew the nature of the discovered particle. Various alternative perspectives to the SM Higgs Boson were still advocated for as late as 2012. For example, perspectives in which the Higgs was seen as a composite particle were still supported by a remarkable number of theorists even post discovery (see Mättig and Stöltzner 2019). What can be suggested is that the discovery precipitated increasing perspectival development on the nature of the discovered Higgs-like particle which led to "growing evidence that the newly discovered particle has properties consistent with the SM expectations" (Mättig and Stöltzner 2019, p. 93) which convinced physicists that it can be accepted "at least tentatively, [as] a SM Higgs boson" (id.). ${ }^{27}$

A similar development can be observed in the case of the positron and of stellar spectra. Briefly, whilst the positron was predicted by P.A.M. Dirac in 1931 and discovered by C.D. Anderson in August 1932, it was not authenticated till at least 1933 (see Creţu 2020). Two main perspectives emerged post-authentication: the hole-theoretic perspective and the field-theoretic perspective. The latter proved more successful, whilst the former was eventually abandoned.

As regards the classification of stars, it is plausible to suggest that the authentication of the spectra was largely ongoing during at least the first two versions of the Henry Draper Catalogue. This is because the first catalogue focussed primarily on the strength and width of the spectral lines, whilst Antonia Maury noticed further peculiarities in the shapes of the lines such as flutedness and haziness. A number of different perspectives were developed after the authentication of stellar spectra. Some astronomers argued that the spectra reveal different evolutionary stages of a star (e.g. Antonia Maury and Ejnar Hertzsprung), whilst others took the spectra as offering clear information about the constitution of stars. ${ }^{28}$

What these examples show is that the two conceptual stages, authentication and perspectival development, play very different roles in the development and evolution of a scientific classification. Authentication is necessary for the theoretical and empirical validation of the phenomena, that is, for establishing that the phenomena is; whilst perspectival development is necessary for establishing the precise nature of the phenomena, understanding what it is. In the next section we'll take a closer look at the impact of scale-relativity on scientific classifications.

\footnotetext{
27 For an in-depth philosophical exploration of the Higgs search and discovery see the 2017 Synthese Special Issue: A philosophical look at the discovery the Higgs boson edited by Richard Dawid, see Dawid (2017).

28 Even amongst those who took spectra to reveal different evolutionary stages of a star, there was some disagreement with regard to which evolutionary stages do the different spectra reflect. Henri Norris Russell, as opposed to Antonia Maury, believed that red signifies the beginning of a star's life, that the star then warms up and becomes yellow or white, and ultimately cools down to its original red light.
} 


\section{Relational kindhood and scale-relativity}

The aim of this section is to explain how the interplay between the authentication of phenomena and the development of perspectives on their nature gives rise to relational kindhood. Further, it will be shown how scale-relativity complicates relational kindhood. In particular, it will be shown that the principle of scale-relativity has both a methodological and an ontological component, both of which play crucial roles in the shaping and evolution of scientific classifications. Historical and contemporary philosophical lessons, from Whewell (1840a, b) and Ladyman and Ross (2007) respectively, will be brought to light to establish the claim that kindhood is relational, scale-relative, and dynamic.

\subsection{Scale matters}

In their survey of physics and the special sciences, Ladyman and Ross (2007) note that we are now in a position to study events on previously unimaginable spatiotemporal and energetic scales. Physics, for example, studies phenomena that last from anywhere around a tiny fraction of a second to years and decades and can also focus on spatial scales infinitely smaller than the "spatial scales of a millimetre to a few thousand miles" which "are all that have concerned us until recently" (p. 11); astrophysics studies phenomena that similarly can last from a fraction of a second to millions of years; whilst geology "require[s] us to adopt time scales that make all of human history seem like a vanishingly brief event" (p. 11). What these examples point to is the scale-relativity of ontology, where,

[s]cale relativity of ontology is the more daring hypothesis that claims about what (really, mind-independently) exists should be relativized to (real, mindindependent) scales at which nature is measurable (p. 200).

For example, as Ladyman and Ross note "at the quantum scale there are no cats; at scales appropriate for astrophysics there are no mountains" (p. 199). A variation of scale, either temporal, spatial or energetic, may reveal more or less or different kinds of phenomena. A case in point here comes from biology, where "in histories of lineages at small enough temporal scales there is no natural selection, because natural selection requires a substantial minimum number of reproductive events" (p. 203). Whilst Ladyman and Ross (2007) were the first to introduce and explicate the principle of scale-relativity in contemporary literature, they didn't go nearly far enough. ${ }^{29}$ This is on the one hand, due to their restricted focus on spatiotemporal scales and length scales, and on the other hand, due to their explicit focus on the ontological dimension of scale-relativity and contemporary sciences.

However, by combining lessons from modern and contemporary scientific practices with the wisdom of historical lessons more aspects of scale-relativity become salient. For example, by reflecting on Whewell's example of the trees, we come to understand not only that individual trees must be authenticated, but also that enough of them must

29 Bursten (2016) also argued that "philosophical accounts of kindhood should be indexed to the length, time, or energy scales of the entities of scientific investigation" (p. 3). 
be authenticated in order to identify relations amongst them, and hence put the basis of a scientific classification. The basic idea behind this example is that the number or the numerosity scale of entities studied matters more than previously appreciated. ${ }^{30}$ What numerosity entails is that "more is different" (Anderson 1972), and that sometimes mere numerosity can make a dramatic difference (see Ladyman and Wiesner 2020). That is, some phenomena become visible only with an increase in quantity, whilst other phenomena can only occur given enough entities or iterations of relations. Let's explicate this in more detail.

Numerosity concerns the number of entities needed to erect a classification and the classificatory changes that occur with changes in quantities. It thus plays both a foundational role and a developmental role. It was already shown that any classification must begin with the authentication of phenomena. It must now be emphasised that enough entities must be authenticated to put the basis of a classification. This is because in a context where there is only one entity, there are no kinds. Even when two completely different entities have been authenticated, we might still be reluctant to talk about kinds. This is because we may have no reason to suppose that there are other entities similar to the two different entities we have authenticated. However, once we have authenticated three entities, if the third entity turns out to be sufficiently similar to at least one of the existing entities, we can begin to talk about kinds. Once we have thus sorted the three entities into kinds, the next entity we authenticate will be assessed in reference to our already existing entities and kinds. What these considerations suggest is that erecting a classification requires a minimum number of entities. To put it differently kinds themselves only become visible with an increase in the number of authenticated phenomena.

The number of entities authenticated matters not only for separating entities into kinds, but also in their evolution. Depending on how many entities are compared with one another, only certain relations will obtain or become salient. For example, before the discovery of the positron, both charge and mass constituted salient differences between the known existing particles, the electron and the proton. After the discovery of the positron it became clear that particles can have the same mass but opposite charge. The main point to emphasise here is that, an increase or decrease in the original sample of entities authenticated and compared, temporally or spatially, or even energetically, may make more or other relations salient. What this means is that classificatory schemes can change more or less dramatically with an increase or decrease in the numerical scale. It is thus unsurprising that scientific classifications are numerosity relative.

That the originally authenticated relations can modify over time, space, or energy scale with an increase or decrease of relevant entities, further points to the interaction between different scales. These interactions, coupled with the ontological scale-relativity of entities and relations themselves, bring into focus the methodological difficulty of keeping track of relational kindhood. This is where perspectives come in. Perspectives are thus not only responsible for fine-grained investigations into the

30 Ladyman and Wiesner (2020) introduce numerosity in complex science. The concept is borrowed without assuming that kinds are complex systems, though this hypothesis may yet lead to further productive ideas about kindhood. 
nature of entities and relations, they are also responsible for navigating the methodological complexity and scale-relativity of scientific classifications.

The interplay between the methodological and the ontological components of scalerelativity can sometimes mask the perspective-independence of relational kindhood. Whewellian lessons can nevertheless help to illustrate in what way the interplay between the authentication of phenomena and the application of classificatory principles for their hierarchical organisation preserves the perspective-independence of authenticated phenomena. At the same time, such considerations also serve to highlight the limitations of the type of ontological commitment compatible with the proposed account.

As Whewell remarks, singling out entities, comparing them, grading observed likeness and differences is a laborious process that involves on the one hand assumptions and on the other hand unintermitting observations of empirical regularities. Both are necessary, for without assumptions or without any empirical regularities, we would not be able to distinguish phenomena from one another and arrange them in hierarchies or be able to study their nature. As Bondi (1955) points out, the privileging of some form of theory-neutral observation "seems to be the result of a deep human prejudice, that if only one continues to look at an object for long enough its nature will become apparent. In science this is, of course, nonsensical. One could stare at a piece of wood for years if not generations without discovering its atomic nature, or being able to infer its properties in any way from appearances" (p. 160). What this points to is the back and forth between empirical observations and assumptions and organising principles involved in any process of classification. This is because the more entities and relations are observed the more laborious the process of organising likenesses and differences in a way that is exact, precise, and empirically adequate becomes. The more laborious the organisation, the more contrived it seems. However, the process is always the same, involving assumptions and entities and relations equally. As more observations become available, the same process is carried out at increasingly larger scales. Just as Whewell notes, the process repeats itself as follows: "[a]s individuals by their resemblances form kinds, so kinds of entities, though different, may resemble each other so as to be again associated in a higher class; and there may be several successive steps of such a classification" (p. 457).

\subsection{Perspective independence}

It is true that both of these acts "of singling out one entity and of finding relations amongst many" are "operations of the mind", which makes it seem that any result of these operations would itself be an operation of the mind. To the extent to which one accepts perspective-independence, one would, however, not draw such a parochial conclusion. Instead, one would see that neither operation is purely an operation of the mind. Both operations are grounded in repeated observations of empirical regularities. On the one hand, repeated observations of trees in forests, and in isolation, warrant the belief that "assertions concerning the object shall be possible" (p. 452). On the other hand, repeated observations of trees would eventually lead one to notice that likeness in the shape of leaves is far more common than likeness in the shape of the branches. 
Thus, on the basis of such observations, the belief that "general assertions shall be possible" (p. 454) is also warranted. What these assumptions signify is that entities and relations are robust beyond their authentication, that is, that they are projectible on a given scale, though may not be projectible on another scale. For example, even the 'authentication' of a single tree in an aspen grove cannot be discarded as outright erroneous on all scales. After all, it is plausible to suggest that its authentication proceeded on a scale on which the Aspen is a robust (tree-like) phenomena, but once further investigation into its nature was undertaken and its root system was studied further, it became clear that the Aspen is not in fact a tree but a grove, and perhaps should be properly investigated on a different scale. It is important to note that the above assumptions are not infallible and that they are warranted to the same degree as induction is warranted. Finally, it is worth emphasising that the two operations described are at the same time based on assumptions as well as on "[ $\mathrm{t}] \mathrm{he}$ unintermitting stream of experience [which] supplies us with an incalculable amount of such observed connexions" (p. 455).

This way of understanding the emergence and evolution of scientific classifications marks the present account as truly situated or perspectival. Chang (2016) drew similar conclusions based on the history of chemical kinds, whilst Reydon (2016) reached a similar conclusion in the case of genes classification. However, whilst both authors acknowledge the situated nature of scientific classifications, they attribute this to the epistemic role classifications play in scientific inquiry. This, however, as we have explained at length here is only one aspect of the situatedness of classifications.

It is not solely the contribution of background assumptions and principles of classification that limit the scope of a classification; the scale-relativity of classifications and the methodological complexity of navigating the interaction of scales, dictate the limited and changing character of a scientific classification. For example, classifications can change with an increase or decrease of entities on particular scales. Thus, as Bursten (2016) similarly notes, "it is a scale-dependence in the systems themselves that provide opportunities and support for scale-dependent changes in the landscape of kinds in a lab" (p. 3). The more general conclusion to draw here is that to understand how classifications work and evolve it is not sufficient to acknowledge the mutual contribution of nature on the one hand and of background assumptions and principles of classification on the other hand. It is equally important to acknowledge the specific, fine-grained 'contributions' of the world itself and the specific limitations dictated by different scales and by different domains of inquiry.

It will be instructive at this point to clarify the ontology entailed by the present account. To do this, a few distinctions are in order. It is standardly assumed that natural kinds must be mind-independent and objective. These notions are not, however equivalent, though often objectivity and mind-independence are conflated. It is not surprising that the two notions are conflated, since objectivity is necessary, but not sufficient for mind-independence. At the same time, mind-independence is not necessary for objectivity. To get a clear grasp on objectivity and mind-independence, further distinctions are called for. ${ }^{31}$

\footnotetext{
31 See also Khalidi (2016)'s paper for a four-tier distinction between types of mind-dependence, as well as Franklin-Hall (2015)'s paper, who distinguishes between 'fully objective' and 'fully mind-independent kinds'.
} 
A classification can be said to be objective when it is unambiguous and intersubjectively well-founded. ${ }^{32}$ Objectivity is a methodological notion, pertaining to the practice of science and not to the nature of the world. ${ }^{33}$ A classification can be objective without being mind-independent, for example the classification of flags. Flags, and what they represent in different circumstances, are human constructs whose ongoing existence and function is dependent upon humans maintaining such constructs and their ongoing performance (Thomasson 2014). In contrast, when a classification is assumed to be mind-independent, it must be independent of human thought. ${ }^{34}$

However, since no inquiry can proceed without at least some theoretical assumptions about the nature of the world-e.g., that the world is structured, that it is stable enough, that there are hierarchies of entities etc. ${ }^{35}$ - complete independence from human thought is impossible to attain. What can be attained, as per ontogenetic considerations and Whewellian historical lessons, are scientific classifications which emerge with empirically driven authenticated relations and entities and evolve with the development of perspectives on the nature of authenticated entities and relations which are continuously informed and reinforced through unintermitting observations of scalerelative empirical phenomena. Thus, insofar as authentication is empirically driven and perspective-less, a tenable, albeit situated, ontological commitment to scale-relative entities and relations becomes available.

The present account, with its commitment to dynamic and scale-relative relational kindhood can be placed squarely alongside Ladyman and Ross's (2007) real patterns based account of natural kinds but it can also be placed within the company of some version of Boyd's (1991, 1999a, 1999b) homeostatic property cluster kinds stripped of its essentialist connotations ${ }^{36}$ as well as cognate naturalistic accounts such as Magnus's (2012), Massimi's (2014), and Slater's (2015). The present account can also be situated within the 'practice and history oriented shift', most recently exemplified in the various proposals found in Kendig (2016b), and stemming from earlier work in a range of fields begun in mid-70's (see Soler et al. 2014). Importantly, the methodology offered here with its fine-grained two stage distinction and its discussion of scale-relativity, particularly numerosity, may lead to fruitful elaborations and improvements for any natural kinds account that has yet to recognise the significance of scale-relativity and ontogenetic considerations.

To sum up, scientific classifications are designed and sustained through the continuous back and forth between empirical observations of entities and relations and the refinement of principles of classification to maintain and increase the success of

\footnotetext{
32 Giere (2006) similarly defines objectivity as "reliable intersubjective agreement", p. 34.

33 Feminist critiques of science have significantly shaped the way we view objectivity in relation to scientific research. Of particular note are the works of Longino (1990), Harding (1991), and Douglas (2009). Daston and Galison (2007) have also offered a historicised conception of objectivity, which significantly influenced the present account.

34 This notion of mind-independence has already been criticised by perspectival realists, see Giere (2006), Massimi (2018a,b). See also Ereshefsky (2018) and Khalidi (2016) for recent discussions on realism and mind-(in)dependence.

35 See fn. 12. See also Massimi (2014), Haslanger (2016), Kendig (2016a).

36 Whilst many authors read Boyd's homeostatic property cluster kinds in broadly realist non-essentialist terms, a few authors have identified hidden essentialist assumptions in Boyd's account. See for example, Griffiths (1999), Massimi (2014), and Slater (2015).
} 
predictions retrodictions, or explanations that the relevant classification sustains. Such classifications are inherently situated due to authentication, as well as the interactions between different scales, both ontologically and methodologically. The commitment to authenticated entities and relations, without which scientific classifications neither emerge nor evolve, constitutes a bone fide, achievable ontological commitment. ${ }^{37}$

\section{Conclusion}

Scientific classifications are invaluable for understanding what entities there are and have been in the world and how they relate to one another. They are equally invaluable for facilitating all forms of epistemic endeavours such as explaining the nature of various phenomena, or making a variety of predictions and retrodictions. Scientific classifications are thus as much about a world of phenomena independent of us as they are about our relation to those phenomena. It was shown that a scale-relative, relational, and dynamic approach to kindhood, informed by both science and the history of science, can help us acquire a better methodology and retain a bone fide ontology. Without examining the science and the history we might fail to notice the dynamic and scale-relative dimensions of our classifications. We might fail to take into account the effects of time, space, energy, numerosity, and perhaps other scales, on our interaction with and understanding of the world around us. The approach developed in this paper motivates a renewed focus on the science and the history of scientific classifications with a particular focus on the stages of their development and their scale-relativity.

Open Access This article is licensed under a Creative Commons Attribution 4.0 International License, which permits use, sharing, adaptation, distribution and reproduction in any medium or format, as long as you give appropriate credit to the original author(s) and the source, provide a link to the Creative Commons licence, and indicate if changes were made. The images or other third party material in this article are included in the article's Creative Commons licence, unless indicated otherwise in a credit line to the material. If material is not included in the article's Creative Commons licence and your intended use is not permitted by statutory regulation or exceeds the permitted use, you will need to obtain permission directly from the copyright holder. To view a copy of this licence, visit http://creativecommons.org/licenses/by/4.0/.

\section{References}

Ackermann, R. J. (1985). Data, instruments, and theory. A dialectical approach to understanding science Princeton University Press.

Anderson, P. W. (1972). More is different. Science, 177(4047), 393-396.

Ball, P. (2019). Extreme chemistry: Experiments at the edge of the periodic table. Nature, 565(7737), $552-556$.

Batterman, R. W., \& Green, S. (2020). Steel and Bone: Mesoscale modeling and middle-out strategies in physics and biology. Synthese, 199(1-2), 1159-1184.

37 Granted, this commitment may not satisfy a host of so-called 'strong' realists, such as Ellis (2001) or Bird (2007) and Hawley and Bird (2011), which require nothing short of an unmitigated mind-independent commitment to hierarchies in nature for kinds to be real, but so-called 'weak' realists should find it relatively uncontroversial. 
Bird, A. (2007). Nature's metaphysics: laws and properties. Clarendon Press.

Bird, A., \& Tobin, E. (2017). Natural kinds. In E. N. Z. (Ed.), The Stanford Encyclopedia of philosophy, Metaphysics Research Lab, Stanford University, spring 2017 edition.

Bondi, H. (1955). Fact and inference in theory and in observation. Vistas in Astronomy, 1, 155-162.

Boyd, R. (1991). Realism, anti-foundationalism and the enthusiasm for natural kinds. Philosophical studies, 61(1), 127-148.

Boyd, R. (1999a). Homeostasis, species, and higher taxa. In R. Wilson (Ed.), Species: New interdisciplinary essays (pp. 141-186). MIT Press.

Boyd, R. (1999b). Kinds as the 'workmanship of men': realism, constructivism, and natural kinds. Rationalität, Realismus, Revision: Vorträge des, 3, 52-89.

Brown, L. M. (1978). The Idea of the Neutrino. Physics Today, 31(9), 23.

Brumfiel, G. (2012). Neutrinos not faster than light. Nature News. https://doi.org/10.1038/nature.2012. 10249

Bueno, O., Chen, R., \& Fagan, M. B. (2018). Individuation, process, and scientific practices. Oxford University Press.

Bursten, J. R. (2016). Smaller than a breadbox: Scale and natural kinds. The British Journal for the Philosophy of Science, 69, 1-23.

Cannon, A. J., \& Pickering, E. C. (1901). 'Spectra of bright southern stars', Annals of The Astronomical Observatory of Harvard College. XXVIII. - Part, II, 129-263.

Cartwright, N. (1983). How the laws of physics lie. Oxford University Press.

Caulton, A. (2013). Discerning "indistinguishable" quantum systems. Philosophy of Science, 80(1), 49-72.

Chang, H. (2016). The rising of chemical natural kinds through epistemic iteration. In C. Kendig (Ed.), Natural kinds and classification in scientific practice (pp. 33-46). Routledge.

Clarke, E. (2010). The problem of biological individuality. Biological Theory, 5(4), 312-325.

Clarke, E. (2012). Plant individuality: a solution to the demographer's dilemma. Biology \& Philosophy, 27(3), 321-361.

Creţu, A. (2020). Diagnosing Disagreements: The Authentication of the Positron 1931-1934. Studies in History and Philosophy of Science Part B: Studies in History and Philosophy of Modern Physics, 70, 28-38.

Creţu, A. (2021). Perspectval instruments. Philosophy of Science. http://philsci-archive.pitt.edu/18949/ (forthcoming).

Darrigol, O. (1988). The quantum electrodynamical analogy in early nuclear theory or the roots of Yukawa's theory. Revue d'histoire des Sciences, 41(3), 225-297.

Daston, L. \& Galison, P. (2007). Objectivity, Zone Books. New York, 2010 (paperback) edition.

Dawid, R. (2015). Higgs discovery and the look elsewhere effect. Philosophy of Science, 82(1), 76-96.

Dawid, R. (2017). A philosophical look at the discovery of the Higgs boson. Synthese, 194, 253-257.

Dennett, D. C. (1991). Real patterns. The Journal of Philosophy, 88(1), 27-51.

Douglas, H. E. (2009). Science, policy, and the value-free ideal. University of Pittsburgh Press.

Ellis, B. (2001). Scientific essentialism. Cambridge University Press.

Ereshefsky, M. (2018). Natural kinds, mind independence, and defeasibility. Philosophy of Science, 85(5), 845-856.

Esfeld, M., \& Lam, V. (2008). Moderate structural realism about space-time. Synthese, 160(1), $27-46$.

Feest, U. (2011). What exactly is stabilized when phenomena are stabilized? Synthese, 182(1), 57-71.

Franklin, A. (1986). The Neglect of experiment. Cambridge University Press, first paperback edition 1989 edition.

Franklin, A. (2017). The missing piece of the puzzle: The discovery of the Higgs boson. Synthese, 194(2), 259-274.

Franklin-Hall, L. R. (2015). Natural kinds as categorical bottlenecks. Philosophical Studies, 172(4), 925948.

French, S. (2019). Identity and individuality in quantum theory. In E. N. Zalta (Ed.), The Stanford encyclopedia of philosophy, https://plato.stanford.edu/archives/win2019/entries/qt-idind/. Metaphysics Research Lab, Stanford University, winter 2019 edition.

Galison, P. (1997). Image and logic: A material culture of microphysics. University of Chicago Press.

Giere, R. N. (2006). Scientific perspectivism. University of Chicago Press, paperback edition 2010 edition.

Green, S. F. \& Jones, M. H. (2015). An introduction to the sun and stars (2nd edn). Cambridge University Press 
Griffiths, P. E. (1999). Squaring the circle: Natural kinds with historical essences. Species: New interdisciplinary essays, 209-28.

Hacking, I. (1983). Representing and intervening. Cambridge University Press.

Hanson, N. R. (1961). Discovering the positron (i). British Journal for the Philosophy of Science 194-214.

Hanson, N. R. (1962). Discovering the positron (ii). British Journal for the Philosophy of Science, $299-313$.

Harding, S. G. (1991). Whose science? Whose knowledge?: Thinking from women's lives. Cornell University Press.

Haslanger, S. (2016) Theorizing with a purpose. In C. Kendig (Ed.), Natural kinds and classification in scientific practice (pp. 129-145). Routledge.

Hawley, K., \& Bird, A. (2011). What are natural kinds? Philosophical Perspectives, 25(1), $205-221$.

Hendry, R. F. (2021). Structure, scale and emergence. Studies in History and Philosophy of Science Part A, $85,44-53$

Hernandez, P. (2016). Neutrino physics. In Proceedings of the 2015 CERN-Latin-American School of High-Energy Physics (Vol. 5).

Hoefer, C. \& Martì, G. (2020). Realism, reference \& perspective. European Journal for Philosophy of Science, 10(38).

Hoffleit, D. (1991). The evolution of the Henry Draper Memorial. Vistas in Astronomy, 34, 107-162.

Kajita, T. (2015). Discovery of atmospheric neutrino oscillations. Nobel Lecture. NobelPrize.org. Nobel Prize Outreach AB 2022. https://www.nobelprize.org/prizes/physics/2015/kajita/lecture

Kant, I. (1781). The critique of pure reason. Cambridge University Press, 1998 (paperback) edition.

Kearney, M. (2007). Philosophy and phylogenetics: Historical and current connections. In D. L. Hull \& M. Ruse (Eds.), The Cambridge companion to the philosophy of biology. Cambridge University Press.

Kendig, C. (2016a). Activities of kinding in scientitic practice. In C. Kendig (Ed.), Natural kinds and classification in scientific practice (pp. 1-13). Routledge.

Kendig, C. (Ed.) (2016b). Natural kinds and classification in scientific practice. Routledge.

Khalidi, M. A. (2016). Mind-dependent kinds. Journal of Social Ontology, 2(2), 223-246.

Kragh, H. (2013). Superheavy elements and the upper limit of the periodic table: Early speculations. The European Physical Journal H, 38(3), 411-431.

Kragh, H. (2017). On the ontology of superheavy elements. Substantia, 1(2), 7-17. https://doi.org/10.13128/ substantia-25.

Kragh, H. (2018). From transuranic to superheavy elements: A story of dispute and creation. Springer.

Kragh, H. (2019). The periodic system and the idea of a chemical element: From Mendeleev to superheavy elements. Centaurus, 61(4), 329-344.

Ladyman, J. (2007). Scientific structuralism: On the identity and diversity of objects in a structure. In Aristotelian Society Supplementary Volume (Vol. 81, pp. 23-43). Wiley Online Library.

Ladyman, J. (2015). Are there individuals in physics, and if so what are they? In A. Guay \& T. Pradeu (Eds.), Individuals Across the Sciences (pp. 193-206). Oxford University Press.

Ladyman, J., \& Ross, D. (2007). Every thing must go. Oxford University Press.

Ladyman, J., \& Wiesner, K. (2020). What is a complex system? Yale University Press.

Laudan, L. (1977). Progress and its Problems: Towards a theory of scientific growth. University of California Press of California Press, 1978 edition.

Longino, H. E. (1990). Science as social knowledge. Values and objectivity in scientific inquiry. Princeton University Press.

Magnus, P. D. (2012). Scientific enquiry and natural kinds: From planets to Mallards. Springer.

Massimi, M. (2014). Natural kinds and naturalised kantianism. Noûs, 48(3), 416-449.

Massimi, M. (2018a). Four kinds of perspectival truth. Philosophy and Phenomenological Research, 96(2), 342-359.

Massimi, M. (2018b). Perspectivism. In J. Saatsi (Ed.), The Routledge handbook of scientific realism (chap. 13, pp. 164-175). Routledge.

Mättig, P., \& Stöltzner, M. (2019). Model choice and crucial tests. On the empirical epistemology of the Higgs discovery. Studies in History and Philosophy of Science Part B: Studies in History and Philosophy of Modern Physics, 65, 73-96.

Mitton, J. B., \& Grant, M. C. (1996). Genetic variation and the natural history of quaking aspen. Bioscience, 46(1), 25-31.

Moreno, L. F. (2019). Reflection on natural kinds. Introduction to the special issue on natural kinds: Language, science, and metaphysics. Synthese, 198(Suppl 12), 2853-2862. 
Muller, F. A., \& Saunders, S. (2008). Discerning fermions. The British Journal for the Philosophy of Science, 59(3), 499-548.

Okasha, S. (2002). Darwinian metaphysics: Species and the question of essentialism. Synthese, 131(2), $191-213$.

Pooley, O. (2006). Points, particles, and structural realism. In D. Rickles, S. French, \& J. T. Saatsi (Eds.), The Structural Foundations of Quantum Gravity (pp. 83-120). Oxford University Press.

Rayner, M. (2020). Tuning in to neutrinos. Cern Courier. https://cerncourier.com/a/tuning-in-to-neutrinos/.

Reich, E. S. (2011). Faster-than-light neutrinos face time trial. Nature. https://doi.org/10.1038/news.2011. 575

Reydon, T. (2016). From a zooming-in model to a co-creation model. In C. Kendig (Ed.), Natural kinds and classification in scientific practice (pp. 59-73). Routledge.

Richards, R. A. (2016). Biological classification. Cambridge University Press.

Roqué, X. (1997). The manufacture of the positron. Studies in History and Philosophy of Science Part B: Studies in History and Philosophy of Modern Physics, 28(1), 73-129.

Ross, D. (1995). Real patterns and the ontological foundations of microeconomics. Economics and Philosophy, 11(01), 113-136.

Russell, B. (1911). On the relations of universals and particulars. Proceedings of the Aristotelian Society, $12,1-24$.

Russell, H. N. (1919). Edward Charles Pickering. Science, New Series, 49(1259), 151-155.

Scerri, E. R. (1998). The evolution of the periodic system. Scientific American, 279(3), 78-83.

Scerri, E. R. (2019). The periodic table: Its story and its significance. Oxford University Press.

Slater, M. H. (2015). Natural kindness. The British Journal for the Philosophy of Science, 66(2), 375-411.

Sober, E. (2000). Philosophy of biology (2nd ed.). Westview Press.

Soler, L., Zwart, S., Lynch, M., \& Israel-Jost, V. (2014). Science after the practice turn in the philosophy, history, and social studies of science. Routledge.

Stachel, J. (2006). Structure, individuality, and quantum gravity. In D. Rickles, S. French, \& J. Saatsi (Eds.), The structural foundations of quantum gravity (pp. 53-82). Oxford University Press.

Thomasson, A. L. (2014). Public artifacts, intentions, and norms. In M. Franssen, et. al. (Eds.) Artefact kinds: Ontology and the human-made world (pp. 45-62). Springer International Publishing.

Wallace, D. (2010). Decoherence and ontology (or: How I learned to stop worrying and love FAPP). In S. Saunders, J. Barrett, A. Kent, \& D. Wallace (Eds.), Many worlds? Everett, quanum theory, and reality (pp. 53-72). Oxford University Press.

Whewell, W. (1837a). History of the inductive sciences (Vol. 1). Cambridge University Press, 2010 (paperback) edition.

Whewell, W. (1837b). History of the inductive sciences (Vol. 2). Cambridge University Press, 2010 (paperback) edition.

Whewell, W. (1837c). History of the inductive sciences (Vol. 3). Cambridge University Press, 2010 (paperback) edition.

Whewell, W. (1840a). The philosophy of the inductive sciences founded upon their history (Vol. 1). Cambridge University Press, 2014 (paperback) edition.

Whewell, W. (1840b). The philosophy of the inductive sciences founded upon their history (Vol. 2). Cambridge University Press, 2014 (paperback) edition.

Wilson, M. (2017). The Greediness of scales. In Physics avoidance. Essays in conceptual strategy. Oxford University Press.

Zeilik, M., \& Gregory, S. (1998). Introductory astronomy and astrophysics. Brooks/Cole.

Publisher's Note Springer Nature remains neutral with regard to jurisdictional claims in published maps and institutional affiliations. 